\title{
Evaluation of the Causes of Cancellation of Cases on the Day of Surgery
}

\author{
Geeta Karki ${ }^{1}$, Vishwadeep Singh ${ }^{2}$, Ashita Mowar ${ }^{2}$ \\ ${ }^{1}$ Associate Professor, Department of Anaesthesiology, SRMS IMS, Bareilly (UP), India, ${ }^{2}$ Assistant Professor, Department of Anaesthesiology, SRMS IMS, \\ Bareilly (UP), India.
}

\section{Abstract}

Background: Cancellation of elective operation on the day of surgery is a common phenomenon in all hospitals, which is a great concern in countries like India with limited resources. This study was aimed to assess incidence and causes for this which will help to enhance efficiency and minimize wastage of resources and manpower. Subjects and Methods: The study was carried out over six months and included all patients posted for elective surgery. The reasons for cancellation were classified into four types - anaesthesia related, patient related, surgeon related and hospital/resource related. Statistical analysis: Descriptive and basic analytical statistics were used and data is expressed as number and percentage. Results: A total of 4447 patients were posted for surgery during the study period of which 595 patients were cancelled on the day of surgery with a cancellation rate of $13.37 \%$. Highest cancellation rate was found in Orthopaedics (39.65\%) followed by ENT (14.32\%), General Surgery (10.02\%) and Obstetrics and Gynaecology (8.53\%). Maximum cancellations were due to surgeon related factors (46.72\%) followed by anaesthesia related (21.68\%), patient related (20\%) and hospital/resource related (11.6\%). Conclusion: Most of the reasons for cancellation are potentially avoidable. If causes are identified and appropriately addressed to the efficiency of OT can be improved. Regular audits are regularly recommended.

Keywords: Elective operation, Cancellation, Anaesthesia, Patient, Hospital, Resource.

Corresponding Author: Dr. Vishwadeep Singh, Assistant Professor, Department of Anaesthesiology, SRMS IMS, Bareilly (UP), India. Email: krkgits@gmail.com

Received: January 2020

Accepted: January 2020

\section{Introduction}

The operation theatre is one of those places of a hospital requiring considerable human resources and expenditure from the hospital budget. However, in developing countries where resources are limited, cancellation of elective surgical operations due to various preventable reasons is a common phenomenon in most hospitals. ${ }^{[1]}$ Cancellation of elective surgery on the scheduled day of surgery (DOS) is defined as when a patient's name has appeared on the scheduled surgery list for the day but the surgery was cancelled on the intended day due to various reasons. DOS cancellation is a worldwide problem, ranging from $0.37-28 \%$ in developed and from 11 to $44 \%$ in developing countries. ${ }^{[2]}$ The rate of surgical cancellation is one of the most important quality indicators of operation theatre facilities. The reasons for cancellations can be patient related, workup related, surgeon related or administrative. ${ }^{[3]}$

Cancellation of elective surgery decrease the over-all efficiency of the operating rooms (ORs), reduces utilization of OR time and waste resource, subsequently endup with high economic burden for the patients and hospitals associated with extended hospital stay and repeated preoperative preparations. Hence, OR generates the highest costs and the largest source of revenues for the hospitals. It affects surgeon productivity and staff morale and also causes psychological trauma or distress for the patients and as well the families.

There are different reasons of cancellation of elective surgery that varies from one hospital to another. The range of reasons given include inadequate pre-op assessment and preparation, management related factors or infrastructural limitations, lack of operating room time, and unavailability of hospital beds, patient-related factors, surgery related factors (surgeon related issues, improper scheduling and Anesthesia related factors. ${ }^{[2]}$

Therefore, this study was aimed to assess incidence and causes of cancellation of elective operation on the intended day of surgery at a tertiary care teaching hospital. The finding of this study will help us to enhance efficiency and minimize wastage of hospital resources and manpower.

\section{Subjects and Methods}

This was a six months prospective hospital-based study which was carried out in the department of Anaesthesiology in from September 2018 to March 2019 in a tertiary care teaching hospital, with a bed capacity of 950 beds, for inpatients of different medical subspecialties. There are 14 
ORs allocated as 5 ORs for GS/week, 2 for orthopedics, 2 for obstetrics \& gynaecology,2 for urology, 1 for ENT, 1 each for plastic surgery and neurosurgery.

The study subjects included all patients, of all ages, both genders, who were scheduled for elective surgical operations except those posted as emergency surgery, Lifesaving surgery and minor/ ambulatory surgery.

The data regarding the elective surgical cases posted for the day were obtained from the operation theatre list prepared on previous day by $4 \mathrm{pm}$. The data regarding cases cancelled on a particular day were obtained from another list that was prepared the day after by 10 am which contained the details of all the cases done / cancelled on previous day. From the list the cancelled cases were noted down and reasons for cancellation were asked from the concerned surgeon or resident and recorded.

The reasons for cancellation were classified into one of the four pre-defined categories -

1) Anaesthesia related

2) Patient related

3) Surgeon related and

4) Hospital/Resource related.

Patient related factors which included patient "no show," patient's refusal to sign consent form and death prior to the date of the operation. Anaesthesia related factors included preoperative preparation causes such as the need for further optimization, further investigation, abnormal tests, or change in the treatment plan and facility related factors including lack of OR time, no elective or ICU beds, unavailability of equipment, implants, or staff administrative errors such as incorrect booking, surgery done as an emergency, or conducted elsewhere.

Descriptive and basic analytical statistics were used to summarize the data. As the study did not involve any pharmacological or non-pharmacological intervention so there are no ethical issues, though ethical clearance from the institute committee was taken. The information collected did not include any personal data so individual consent was also not required.

\section{Results}

The study was carried out over a period of six months from September 2018 to February 2019 in the Department of Anaesthesiology of a tertiary care teaching hospital. A list of all patients posted for surgery during the study period was prepared. A list of all patients whose surgery got cancelled was also prepared. A cancellation was defined as the surgery that was scheduled on the final operative list but was not performed subsequently. A total of 4447 patients were scheduled for surgery in the operative list during this period, out of which 595 patients were cancelled on the day of surgery.

The [Table $1 \&$ Figure 1] shows the total number of cases per speciality scheduled for surgery and postponed on day of surgery during this period. In our institute, the various surgical superspecialities are not separate departments but part of surgery department. So in the present study, the cases of superspecialities like paediatric surgery, urosurgery, neurosurgery, oncosurgery, plastic surgey were considered as part of General Surgery. During the study period, a total of 4447 patients were posted for surgery in General Surgery, Orthopaedics, ENT and Obstetrics \& Gynaecology specialities together. Out of a total of 4447 patents, the maximum number of patients were posted in General Surgery $(3233,72.70 \%)$, followed by 469 (10.54\%) patients in Orthopaedics, $375(8.43 \%)$ patients in Obstetrics \& Gynaecology and $370(8.32 \%)$ in ENT. As shown in the table, a total of 4447 patients were posted for surgery during this period. Out of these 595 patients were cancelled with a cancellation rate of $13.37 \%$. In orthopaedics, a total of 469 patients were posted of which 186 were cancelled with a cancellation rate of $39.65 \%$, which was the highest cancellation rate in our study. In ENT, a total of 370 patients were posted of which 53 patients were cancelled on the day of surgey, with the second highest cancellation rate of 14.32 $\%$. In general surgery, a total of 3233 patients were posted, of which 324 were cancelled, with a cancellation rate of $10.02 \%$. In obstetrics \& gynaecology, a total of 375 patients were posted, of which 32 patients were cancelled, with a cancellation rate of $8.53 \%$, the least in our study.

Of the 595 patients cancelled, 324 patients were cancelled in general surgery, accounting for $7.28 \%$ of all the cancellation followed by orthopaedics where 186 patients were cancelled accounting for $4.18 \%$ of the cancellations. A total of 53 patients were cancelled in ENT accounting for $1.19 \%$ of the cancellations. In obstetrics \& gynaecology a total of 32 patients were cancelled accounting for $0.17 \%$ of the cancellations.

Table 1: Total number of cases posted and postponed.

\begin{tabular}{|l|l|l|l|}
\hline & $\begin{array}{l}\text { Cases } \\
\text { posted }\end{array}$ & $\begin{array}{l}\text { Cases } \\
\text { postponed }\end{array}$ & Percentage \\
\hline General surgery & 3233 & 324 & 10.02 \\
\hline Obs \& gynae & 375 & 32 & 8.53 \\
\hline Orthopaedics & 469 & 186 & 39.65 \\
\hline ENT & 370 & 53 & 14.32 \\
\hline Total & 4447 & 595 & 13.37 \\
\hline
\end{tabular}

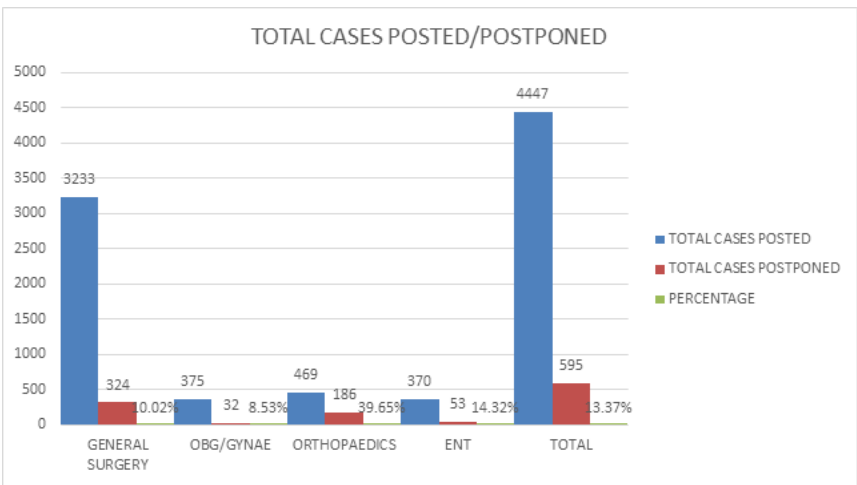

Figure 1: Total number of cases posted and postponed

In our study, the reasons for cancellation were classified into one of the four predefined categories -1) Anaesthesia related 2) Surgeon related 3) Patient related and 4) Hospital/ Resource related.

[Table $2 \&$ Figure 2] shows the number of cases cancelled due to anaesthesia related causes in different specialities. Out of a total of 595 cancelled cases, 129 (21.68\%) cases were cancelled due to anaesthesia related causes. In general surgery, out of 324 patients cancelled, 77 (23.76\%) patients 
were cancelled due to anaesthesia related causes. In orthopaedics, out of 186 patients cancelled 36 (19.35\%) were cancelled due to anaesthesia related causes. In obstetrics \& gynaecology, out of 32 cancelled patients 7 (21.87\%) and in ENT out of 53 patients cancelled $9(16.98 \%)$ were cancelled due to anaesthesia related causes.

Table 2: Cases postponed due to anaesthesia related factors

\begin{tabular}{|c|c|c|c|c|c|}
\hline & $\begin{array}{l}\text { Total Cases } \\
\text { Posted }\end{array}$ & $\begin{array}{l}\text { Total Cases } \\
\text { Postponed }\end{array}$ & $\begin{array}{l}\text { Cases Postponed Due To } \\
\text { Anaesthesia Related Causes }\end{array}$ & $\begin{array}{l}\text { Percentage Of Cases } \\
\text { Postponed Of Total } \\
\text { Cases Posted }\end{array}$ & $\begin{array}{l}\text { Percentage Of Cases Postponed } \\
\text { Due To Anaesthesia Of Total } \\
\text { Postponed Cases }\end{array}$ \\
\hline General surgery & 3233 & 324 & 77 & 2.38 & 23.76 \\
\hline Obs \& gynae & 375 & 32 & 7 & 1.87 & 21.87 \\
\hline Orthopaedics & 469 & 186 & 36 & 7.67 & 19.35 \\
\hline ENT & 370 & 53 & 9 & 2.43 & 16.98 \\
\hline Total & 4447 & 595 & 129 & 2.90 & 21.68 \\
\hline
\end{tabular}

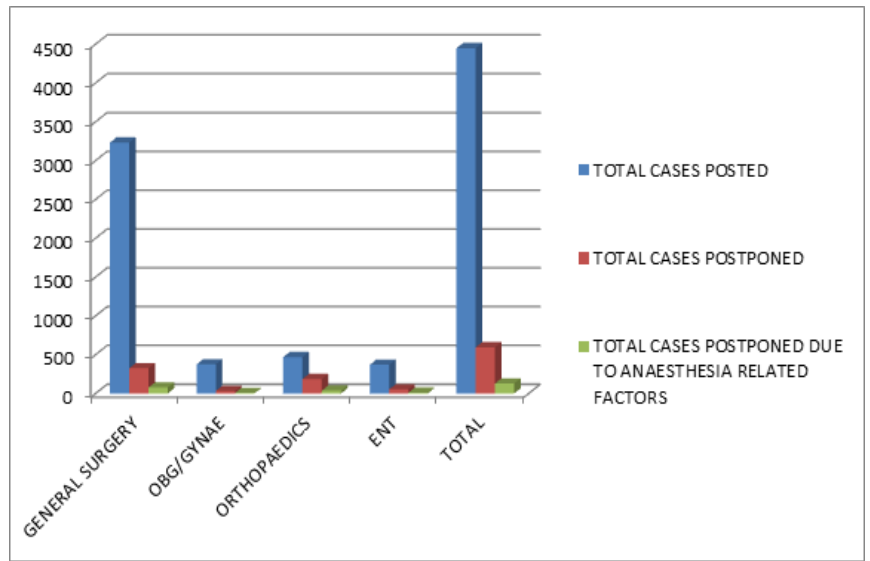

Figure 2: Cases postponed due to anaesthesia related factors

[Table 3 \& Figure 3] shows the number of cases cancelled due to hospital/resource related causes. Out of a total of 595 patients cancelled, $69(11.60 \%)$ patients were cancelled due to hospital/resource related causes. In general surgery 30 (9.26\%) patients, in orthopaedics $25(13.44 \%)$ patients, in obstetrics \& gynaecology $5(15.62 \%)$ patients and in ENT 9 (16.98\%) patients were cancelled due to patient related causes.

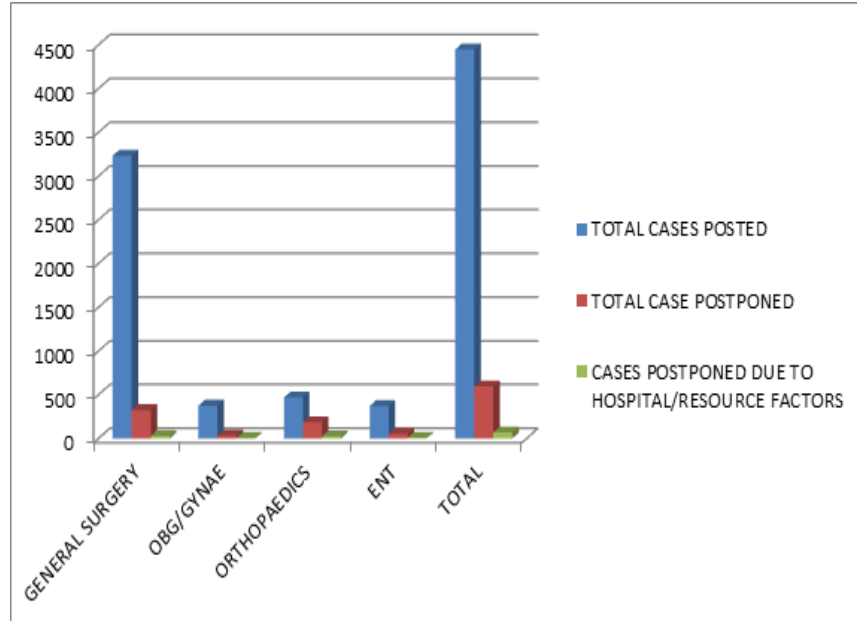

Figure 3: Cases postponed due to hospital / resource related factors

[Table $4 \&$ Figure 4] shows the number of cases cancelled due to patient related causes. Of the 595 cases cancelled, 119 (20\%) cases were cancelled due to patient related causes. In general surgery, $57(17.59 \%)$ patients, in orthopaedics 35 $(18.82 \%)$, in obstetrics \& gynaecology 12 (37.50\%), and in
ENT $15(28.30 \%)$ patients were cancelled due to patient related causes.

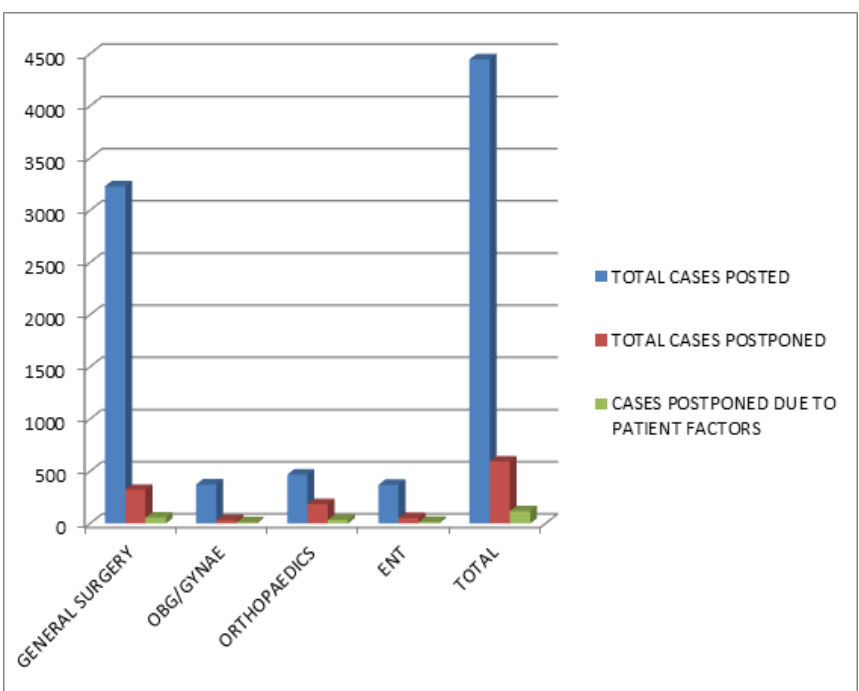

Figure 4: Cases postponed due to patient related factors

[Table $5 \&$ Figure 5] shows the number of cases cancelled due to surgeon related causes in different specialities. A total of 595 cases were cancelled of which 278 (46.72\%) were cancelled due to surgeon related causes.In general surgery, $160(49.38 \%)$ patients were cancelled due to surgeon related causes. In orthopaedics, 90 (48.39\%), in obstetrics \& gynaecology $8(25 \%)$ and in ENT $20(37.73 \%)$ patients were postponed due to surgeon related causes.

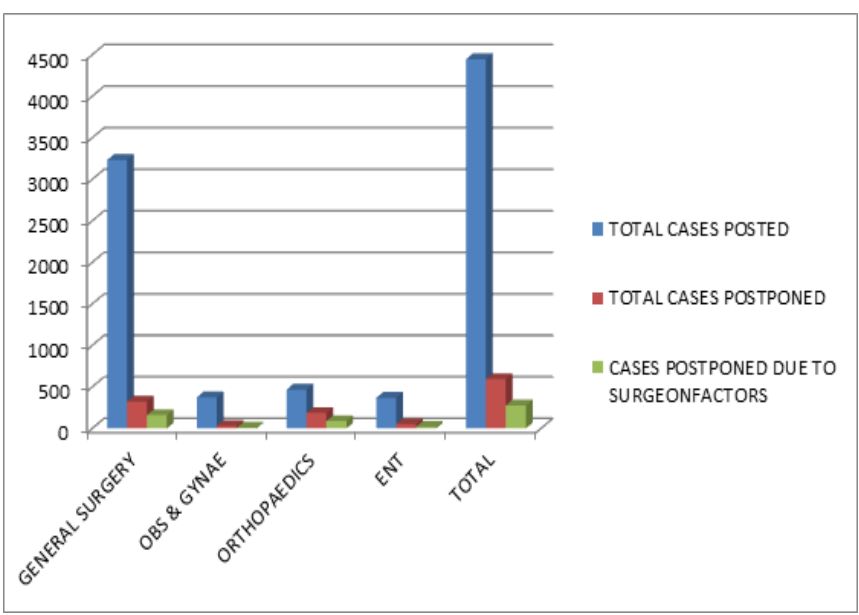

Figure 5: Cases postponed due to surgeon related factors 
Table 3: Cases postponed due to hospital / resource related factors

\begin{tabular}{|l|l|l|l|l|l|}
\hline & $\begin{array}{l}\text { Total cases } \\
\text { posted }\end{array}$ & $\begin{array}{l}\text { Total cases } \\
\text { postponed }\end{array}$ & $\begin{array}{l}\text { Cases postponed due } \\
\text { to hospital/resource }\end{array}$ & $\begin{array}{l}\text { \% cases postponed } \\
\text { of total cases posted }\end{array}$ & $\begin{array}{l}\text { \% cases postponed of total cases } \\
\text { postponed }\end{array}$ \\
\hline General Surgery & 3233 & 324 & 30 & 0.93 & 9.26 \\
\hline Obs \&Gynae & 375 & 32 & 5 & 1.33 & 15.62 \\
\hline Orthopaedics & 469 & 186 & 25 & 5.33 & 13.44 \\
\hline ENT & 370 & 53 & 9 & 2.43 & 16.98 \\
\hline Total & 4447 & 595 & 69 & 1.55 & 11.60 \\
\hline
\end{tabular}

Table 4: Cases postponed due to patient related factors

\begin{tabular}{|l|l|l|l|l|l|}
\hline & $\begin{array}{l}\text { Total Cases } \\
\text { Posted }\end{array}$ & $\begin{array}{l}\text { Total Cases } \\
\text { Postponed }\end{array}$ & $\begin{array}{l}\text { Cases Postponed Due To } \\
\text { Patient Related Causes }\end{array}$ & $\begin{array}{l}\text { \% Cases Postponed Of } \\
\text { Total Cases Posted }\end{array}$ & $\begin{array}{l}\text { \% Cases Postponed Of Total Cases } \\
\text { Postponed }\end{array}$ \\
\hline General surgery & 3233 & 324 & 57 & 1.77 & 17.59 \\
\hline OBS \& Gynae & 375 & 32 & 12 & 3.20 & 37.50 \\
\hline Orthopaedics & 469 & 186 & 35 & 7.46 & 18.82 \\
\hline ENT & 370 & 53 & 15 & 4.05 & 28.30 \\
\hline Total & 4447 & 595 & 119 & 2.67 & 20.00 \\
\hline
\end{tabular}

Table 5: Cases postponed due to surgeon related factors

\begin{tabular}{|l|l|l|l|l|l|}
\hline & $\begin{array}{l}\text { Total Cases } \\
\text { Posted }\end{array}$ & $\begin{array}{l}\text { Total Cases } \\
\text { Postponed }\end{array}$ & $\begin{array}{l}\text { Cases Postponed Due To } \\
\text { Surgeon Related Causes }\end{array}$ & $\begin{array}{l}\text { \% Cases Postponed Of } \\
\text { Total Cases Posted }\end{array}$ & $\begin{array}{l}\text { \% Cases Postponed Of Total Cases } \\
\text { Postponed }\end{array}$ \\
\hline General surgery & 3233 & 324 & 160 & 4.96 & 49.38 \\
\hline OBS \& Gynae & 375 & 32 & 8 & 2.13 & 25.00 \\
\hline Orthopaedics & 469 & 186 & 90 & 19.19 & 48.39 \\
\hline ENT & 370 & 53 & 20 & 5.40 & 37.73 \\
\hline Total & 4447 & 595 & 278 & 6.25 & 46.72 \\
\hline
\end{tabular}

\section{Discussion}

Our institute is a 950 bedded teaching and tertiary care hospital in Uttar Pradesh. The study was carried out in the Department of Anaesthesiology over a period of six months from September 2018 to February 2019. We have a total of 14 operation theatres with surgeries performed of various broad specialities and superspecialities. In our institute, we don't have separate superspeciality departments but instead they are part of General Surgery department. So in our study the surgeries of superspecialities like urosurgery, neurosurgery, oncosurgery, paediatric surgery and plastic surgery are included in General Surgery.

Considerable human and financial resources are invested by the hospital administration into maintaining operation theatres. Last minute cancellation of a scheduled surgery is of great concern. Cancellation of surgery on the day of surgery is a major cause of waste of resources and inefficient use of OT time. It is also potentially stressful with depressing effects and costly to the patient in terms of working days lost and disruption of daily life. ${ }^{[4,5]}$ The last minute cancellation of surgery has not only clinical implications but also psychological, social and financial implications .To provide efficient surgical services, the facility should have a low rate of cancellation. Although there is no consensus on the acceptable rate of case cancellation, when analyzing the efficiency of theatre facilities, less than $5 \%$ is generally recommended. ${ }^{[6]}$

During the study period, a total of 4447 patients were scheduled for surgery. Out of these 595 patients were cancelled on the day of surgery with a cancellation rate of $13.7 \%$. The day of surgery cancellation rate reported in various studies vary widely among institutions from $10-40$ $\%$. The cancellation rate depends on the type of institution, the type of surgery, the population served and the healthcare system. Study conducted by Rakesh Garg et al, ${ }^{[7]}$ in a government hospital in New Delhi observed a cancellation rate of $30.3 \%$ which is higher than that in our study. In a study conducted by Rajender Kumar and Ritika Gandhi ${ }^{[8]}$ in a 500 bedded multispeciality government hospital, the cancellation rate was found to be $17.6 \%$ which is close to that found in our study. In UK, $8 \%$ of scheduled elective operations are cancelled nationally within $24 \mathrm{~h}$ of surgery. ${ }^{[9]}$ Study conducted by Desta et al, ${ }^{[2]}$ at a tertiary referral academic medical center in Ethiopia observed a cancellation rate as high as $31.6 \%$.

A cancellation rate of $11.1 \%$ close to that found in our study was observed by Fayed et al, ${ }^{[10]}$ in a tertiary hospital. A retrospective study conducted by Farhanul Huda3 in a teaching hospital reported a cancellation rate of as low as 6.8\%. Studies in Australia4 and Spain11 found cancellation rates of $11.9 \%$ and $6.5 \%$ respectively.

In our study, the reasons for cancellation were classified into 4 categories according to a predefined criteria.

1) Surgeon related - which included over running of scheduled time, surgeon not available, team involved in another emergency, change of surgical plan, change of diagnosis due to some new finding.

2) Anaesthesia related - Sudden change in medical status, abnormal laboratory tests, Incomplete work up.

3) Patient related - patient not turning up, consent not given, money not deposited, preoperative instructions not followed, blood not arranged.

4) Hospital/Resource related - Non availability of autoclaved instruments/linen, Lack of staff, Equipment failure, non availability of bed in ICU/HDU, disruption of electric / water supply.

In our study maximum number of on day cancellations were due to surgeon related factors $(46.72 \%)$. Surgeon related factors were the commonest cause for cancellation $(35.8 \%)$ in the study conducted by Desta et al, ${ }^{[2]}$ Among surgeon related factors the most common reason was over running of the available time for elective surgery. The reason for overrun of time was either the previous surgery taking more 
time than expected or overbooking of the list. Both of these are potentially avoidable reasons. Several studies have found lack of theatre time to be one of the commonest reason for cancellation of surgery. ${ }^{[4]}$ One study in New Delhi found that lack of time accounted for $59.7 \%$ of the cancellations which is higher than that in our study.7 One study in Pakistan and one in Portugal, ${ }^{[12]}$ also found that lack of time accounted for $36 \%$ and $32 \%$ of the cancellations which is lower than that in our study. ${ }^{[9,12]}$ Literature shows that lack of time is due to late starts, delay in between cases, overrunning of lists and overbooking. ${ }^{[13]}$ Late starts can be avoided by punctuality among surgeons, anaesthetists and staff. Ours is a teaching institute so both anaesthesia and surgery consultants are involved in postgraduate teaching which is held in morning hours and may be the reason of late arrival in OT. Delay in between cases can be due to delay in recovery of patient from anaesthesia though it is not a routine occurrence or due to time taken for cleaning the OT, setting up of anaesthesia equipment, preparation of surgical instrument trolley etc. Overbooking can be reduced by taking into consideration the surgical case duration while preparing the list of booked cases. The time taken for a surgery depends not only on the procedure but also the skill of the surgeon. Ours being a teaching institute, the trainees are also performing procedure which increases the duration of surgery than the expected time. Sometimes the duration may be increased due to some technical difficulties or surgical complications. It is shown in a study that underestimating the surgical time by 10 minutes was associated with a cancellation of $11 \%$ as compared to $6 \%$ cancellation when time was overestimated. ${ }^{[14]}$ Pandit JJ et ${ }^{a l},{ }^{[15]}$ in their study observed that there is a $50 \%$ overbooking of list due to the pressure of waiting patients as well as to present a perception of working hard. Cancellation of surgery due to anaesthesia related reasons was the second most common category of reason for cancellation accounting for $21.68 \%$ of cancellations. Anaesthesia related reasons included any acute change in medical status, abnormal laboratory tests or inadequate work up. A good preoperative assessment and appropriate physician consultation can avoid such cancellations. Improved communication between surgeons, physicians and auxiliary services may expedite preoperative patient evaluation. Dufek et al, ${ }^{[16]}$ recommended improving the timeliness response by consultant physicians, along with improvement of protocol for preoperative patient evaluation as a means of addressing these problems. The study conducted by Farhanul Huda3 observed a cancellation of $16.2 \%$ due to anaesthesia work up related reasons which is lower than our study. In a study conducted by Fayad A et al, ${ }^{[10]}$ the rate of case cancellation due to anaesthesia related reasons was found to be $35.2 \%$ which is much higher than in our study. The timing of the preanaesthesia assessment may influence the cancellation rate on the day of surgery. Although early outpatient preoperative evaluation has been advocated, in actual clinical practice, preoperative evaluation on the day of surgery or the day before surgery is very common and is true for our institute also. Many surgical procedures are delayed or cancelled due to inadequate preoperative assessment and preparation. ${ }^{[17]}$

Patient related reasons accounted for 119 (20\%) cancellations in our study, which included most commonly the patient not turning up, not giving consent, not following instructions and financial issues. It is difficult to establish the reason behind this but a better communication between the patient and the surgical team may help in reducing the cancellations of this type. In the study conducted by Farhanul Huda a cancellation rate of $10 \%$ due to patient related reasons was shown, ${ }^{[3]}$ which is very less as compared to our study. In a study conducted by Fayad A et al, ${ }^{[10]}$ the rate of case cancellation due to patient related reasons was found to be $35.9 \%$ which is much higher than in our study. The study conducted by Desta et al, ${ }^{[2]}$ revealed that patient related factors accounted for $30 \%$ cancellations. Similar results have been found in studies done in Brazil, ${ }^{[18]}$ India and Burkina Faso. ${ }^{[7,19]}$

Out of 595 cases cancelled, 69 (11.6\%) cases were cancelled due to hospital/resource related reasons. These cancellations can be attributed to poor communication and lack of coordination between different departments involved in3. The study conducted by Farhanula Huda, ${ }^{[4]}$ observed a cancellation rate of $29.5 \%$ due to administrative reasons. Administration related factors accounted for $21.2 \%$ of cancellations in the study conducted by Desta et al, ${ }^{[2]}$ which is higher than that in our study.Similar results were found in studies done in China, ${ }^{[20]}$ Saudi Arabia and Sudan In our study, ${ }^{[21,22]}$ the different surgical specialities displayed varied rate of cancellation. The highest rate of cancellation was observed in Orthopaedics $(39.65 \%)$ followed by ENT $(14.32 \%)$, General Surgery $(10.02 \%)$ and Obstetrics \& Gynaecology (8.53\%). In study by Fayad A et al, ${ }^{[10]}$ General Surgery along with Orthopedics displayed the highest rate of cancellation which is similar to that in our study. In the study conducted by Desta et al, ${ }^{[2]}$ also highest percentage $(28.7 \%)$ of cancelled cases was observed in Orthopedics. This finding is also in accordance with other studies. ${ }^{[20,21,23,24]}$ Reasons for cancellation in different specialities also displayed the same trend with surgeon related reasons being the commonest and resource/hospital related reason being the least common. Numerous studies have investigated cancellation of surgery in different surgical specialities and defined similar reasons with varying grades. ${ }^{[20,25]}$ If we closely explore the reasons for cancellation, it will be clear that most of the common reasons for on day cancellation of surgeries are potentially avoidable with meticulous planning, cooperation and communication among administration, surgeon, anaesthetist and patients. It has been reported in literature that more than $50 \%$ of cancellations could be prevented. ${ }^{[4,12,26]}$

\section{Conclusion}

A cancellation rate of $13.7 \%$ in a tertiary care teaching hospital though less than that documented in many studies, is still quite significant. Most of the reasons for cancellation are potentially avoidable. If causes are identified and appropriately addressed to, the efficiency of OT can be improved. Further studies are needed to make recommendations for increasing the efficiency of OT. The on day cancellation of surgery can be reduced by review of patients before surgery and making protocols of patient preparation for various surgeries and strictly adhering to them. Regular audits are highly recommended to assess progress on reduction in cancellations. 


\section{Limitation of Study}

The limitation of our study is that it was done over a short period of duration and we have not calculated the percentage of individual reasons. Further studies over a longer duration of period and study of individual reasons is required.

\section{References}

1. Chalya PL, Gilyoma JM, Mabula JB, et al. Incidence, causes and pattern of cancellation of elective surgical operations in a university teaching hospital in the Lake Zone, Tanzania. Afr Health Sci. 2011;11(3):438-43.

2. Desta M, Manaye A, Abiot. Incidence and causes of cancellation of elective operation on the intended day of surgery at a tertiary referral academic medical center in Ethiopia. Patient safety in Surgery. 2018;12:25.

3. Huda F. A Retrospective analysis of reasons for cancellation of elective surgery in a teaching hospital. Int J Sci Stud. 2014;2(2):28-30.

4. Schofield WN, Rubin G, Pizza M, et al. Cancellation of operations on the day of intended surgery at a major Australian referral hospital. Med J Aus. 2005;182:612-5.

5. Wildner M, Bulstrode C, Spivey J et al. Avoidable causes of cancellation in elective orthopaedic surgery. Health Trends. 1991;23:115-6.

6. Macario A. Are your hospital operating rooms efficient ? A scoring system with eight performance indicators. Anesthesiology. 2006;105:23740.

7. Garg R, Bhalotra AR, Bhadoria P, Gupta N, Anand R. Reasons for cancellation of cases on the day of surgery - A prospective study. Indian $\mathbf{J}$ Anaesth. 2009;53(1):35-39.

8. Kumar R, Gandhi R. Reasons for cancellation of operation on the day of intended surgery in a multidisciplinary 500 bedded hospital. J Anaesthesiol Clin Pharmacol. 2012;28(1):66-69.

9. Zafar A, Mufti TS, Griffin S, Ahmed S, Ansari JS. Cancelled elective general surgical operation in Ayub teaching Hospital. J Ayub Med Coll Abbottabad. 2007;19:64-6.

10. Fayed A, Elkouny A, Zoughaibi N, Wahabi HA. Elective surgery cancellation on day of surgery: An endless dilemma. Saudi J Anaesth 2016; 10:68-73.

11. Gonzalez AA, Gomez Arnau JI, Dela Cruz FJ et al. Causes for cancellation of elective surgical procedures in a Spanish general hospital. Anaesthesia. 2009;64:487-493.

12. Catre D, Carvalho J, Figueiredo E et al. Evidence based Practice and Quality improvement. Eur J Anaesthesiol. 2010;27(47):20.
13. Lebowitz P. Why can't my procedure start on time? AORN J.2003;77(3):594-7.

14. Eijkemans MJ, Van Houdenhoven M, Nguyen T, Boersma E, Steyerberg EW, Kazemier G. Predicting the unpredictable: a new prediction model for operating room times using individual characteristics and the surgeon's estimate. Anesthesiology. 2010;112:41-9.

15. Pandit JJ, Carey A. Estimating the duration of common elective operations: implications for operating list management. Anethesia. 2006;61:768-776.

16. Dufek S, Gaucher E, Gialanella. The total quality process applied to the operating rooms and other clinical processes. Surgery. 1993;113:255-259.

17. Jonnalagadda R, Walrond ER, Hariharan S, et al. Evaluation of the reasons for cancellations and delays of surgical procedures in a developing country. Int J Clin Practice. 2005;59:716-20.

18. Santos GAAC, SCCM B. Cancellation of elective surgeries in a Brazilian public hospital: reasons and estimated reduction. Rev Bras Enferm. 2017;70(3):535-42.

19. Lankoandea M, Bonkoungoub P, Traorea S, Kaborea R, Ouangrec E, Pendeville P. Cancellation of elective surgical procedures in the university teaching hospital center Yalgado Ouedraogo in Burkinia Faso: incidence, reasons and proposals for improvement. South Afr J Anaesth Analg. 2016;22(5):140-4.

20. Chiu Ch, Lee A, Chui PT. Cancellation of elective operations on the day of intended surgery in a Hong Kong hospital: point prevalence and reasons. Hong Kong Med J. 2012;18:5-10.

21. El-Dawlatly A, ATurkistani, Aldohayan A, Zubaidi A, Ahmed A. Reasons of Cancellation of Elective Surgery in a Teaching Hospital. Int J Anesthesiol. 2007;15:2.

22. Mutwali IM, Abbass AM, Elkheir IS, Arbab SS, Bur A, Geregandi T. Cancellation of elective surgical operations in a teaching hospital at Khartoum Bahri, Sudan. Sudan Med Monit. 2016;11(2):196.1.28.51.

23. Laisi J, Tohmo H, Keranen U. Surgery cancellation on the day of surgery in same day admission in a Finnish hospital. Scand J Surg 2013;102:204-

24. Argo JL, Vick CC, Graham LA, Itani KM, Bishop MJ, Hawn MT. Elective surgical case cancellation in the Veterans Health Administration system: Identifying areas for improvement. Am J Surg 2009;198:600-6.

25. Leslie R, Beiko D, Van Vlymen J, Siemens D. Day of surgery cancellation rates in urology: Identification of modifiable factors. Can Urol Assoc J. 2013;7:167-73.

26. Jimenez A, Artigas C, Elia M, et al. Cancellations in ambulatory day surgery: Ten years observational study. J Ambulatory Surg. 2006;12:119. 23.

Copyright: (C) the author(s), 2020. It is an open-access article distributed under the terms of the Creative Commons Attribution License (CC BY 4.0), which permits authors to retain ownership of the copyright for their content, and allow anyone to download, reuse, reprint, modify, distribute and/or copy the content as long as the original authors and source are cited.

How to cite this article: Karki G, Singh V, Mowar A. Evaluation of the Causes of Cancellation of Cases on the Day of Surgery. Acad. Anesthesiol. Int. 2020;5(1):17-22.

DOI: dx.doi.org/10.21276/aan.2020.5.1.4

Source of Support: Nil, Conflict of Interest: None declared. 